\title{
Interstitial lung disease in mixed connective tissue disease
}

\author{
Marshell Tendean', Sazkia Aziza Nuriawan', Pringgodigdo Nugroho²
}

\begin{abstract}
${ }^{1}$ Internal Medicine Department, Faculty Medicine of University of Indonesia/ Cipto Mangunkusumo General Hospital;

${ }^{2}$ Kidney and Hipertension Division, Faculty Medicine of University of Indonesia/ Cipto Mangunkusumo General Hospital
\end{abstract}

Correspondence: Marshell Tendean, MD dr.mars.23@gmail. com

\begin{abstract}
Asbtract
Interstitial lung diseases (ILD) are known as a debilitating pulmonary complications that may be occured in almost all systemic connective tissue diseases (CTD), including mixed connective tissue disease (MCTD). ILD is usually found in more than half of MCTD patients after 2-4 years after the diagnosis made. A-47-years-old female initially diagnosed as systemic lupus erythematosus (SLE) developed a severe progressive dyspnea. She has recently diagnosed as MCTD with ILD after 9 months of initial symptoms. She was giving with Cyclophosphamide $500 \mathrm{mg}$ IV pulse dose. However, after 1 months she developed severe pneumonia and pronounced demise due to intractable septic shock. The debilitating course of ILD is commonly seen in most systemic CTD. Therefore, it is important to perform initial screening and prevention. Systemic corticosteroid with or without immunosupressor agent(s) are indicated in ILD-MCTD. Patients with progressive diseases will have poor prognosis.
\end{abstract}

Keywords : ILD, MCTD, Corticosteroid

\section{Introduction}

Interstitial lung disease (ILD) and pulmonary hypertension $(\mathrm{PH})$ are known as the main cause of mortality and morbidity among patients with Collagen vascular disease (CVD) ${ }^{1,2}$ ILD is a heterogeneous group of non-neoplastic parenchymal lung disorders hallmarked by vary degrees of inflammation and fibrosis that share common radiologic, pathologic, and clinical manifestation. ${ }^{3}$ ILD does not only affect the interstitium but also the airspaces, peripheral airways, and vessels respectively with their epithelial and endothelial linings. ILD, one of CTD manisfestation, seldomly occurs in MCTD. ${ }^{4}$ In CTD, there are two major presentations of ILD; first ILD can be the initial symptoms or become one of the symptoms of CTD ${ }^{4}$ second ILD found as interstitial pneumonia in patients who do not meet the CTD criteria, but later defined as interstitial pneumonia with autoimmune features (IPAF). ${ }^{5}$

Here we present a case of a 47 years old woman with chronic cough, progressive dyspnea, and previous history of SLE and Scleroderma. She was later diagnosed as ILD and CTD due to MCTD and underwent cyclophosphamide IV pulse therapy.

\section{Case llustration}

A 47 years old female refered to our institution due to progressive dyspnea for 2 weeks prior to admission (PTA).

Nine months PTA she complained of intermittent fever. She had non reproductive cough and multiple swollen-tender joints. Yet denied any weakness, abdominal pain, facial erythema, morning stiffness, hair loss, and dysuria. She had given medicines to relive the symptoms, but no relieve were noted yet.

Six months PTA, persistent fever, joint pain and non-productive cough were noted. She developed dyspnea on activity, facial erythema with sun exposure, hair loss, multiple oral ulcers, and weakness notably over her both legs. She also complained morning stiffness persist more than 30 minutes over both arms, and multiple assymetry swollen-tender joints. There were features of scleroderma and Reynauds phenomenon. Further, there were positive anti neutrophilic antibody (ANA), hence diagnosed as systemic lupus erymathosus (SLE) and she got methyl-prednisone (MP) daily $3 \times 4 \mathrm{mg}$ peroral (PO).

Four months PTA, the swollen joints were improved, persistent fever back to intermittent fever. But the other symptoms were persisted. She regularly consulted to her physician and got MP $3 \times 4$ $\mathrm{mg} / \mathrm{PO}$ daily. Further assessment showed moderate positivity of ANA anti-SCL 70, and anti Sm-RNP which brought the diagnosis to scleroderma. So, additional methotrexate (MTX) $1 \times 5 \mathrm{mg} / \mathrm{PO}$ and folic acid $1 \times 5 \mathrm{mg} / \mathrm{PO}$ weekly were given.

Two weeks PTA, she had another episode of high grade fever, cough and dyspnea. She readmitted to hospital due to severe pneumonia for 1 weeks. From the hospital, she was referred to our institution.

In her previous medical history, she has been treated for lymphadenitis tuberculosis 2 times with undocumented treatment. She is non-smoker and non-alcoholic drinker, denies any promiscuities and narcotics. She had a history of previous miscarriage below 18 weeks of gestation on her third pregnancy.

When assesment made, she was alert and had mild respiratory distress. Blood pressure : 90/60 $\mathrm{mmHg}$, Heart rate: 105/min, respiratory rate: 24 cycle/min, temperature: $38^{\circ} \mathrm{C}$. Her face shown a normal facial expression with thickening. There were some area show non scarring hair loss, pale 
palpebral conjunctiva, multiple palatal ulcers and jugular venous pressure noted $5+0 \mathrm{cmH} 2 \mathrm{O}$. Chest and lung assesment showed normal vesicular breath sound with bilateral basal crackles and negative wheezes. There were tenderness over both shoulders, elbows, right knee, and right foot; skin thickening on both arms and feet; periungual ulcers; Reynaud's phenomenon; and vascular telangiectasia over the plantar of both feet.

Laboratory test were performed, such as complete blood count, liver function test, renal function test, coagulation state, electrolytes, metabolic activity, inflammation state, and screening TB, hepatitis $\mathrm{B}$, and hepatitis $\mathrm{C}$ infection. Besides, we also performed analysis for ANA state. Screening for HIV, tuberculosis, hepatitis B and C showed unremarkable results. For the other laboratory results is shown in Table 1. Her electrocardiography (ECG) findings showed sinus tachycardia. No other abnormalities were found. Chest X-ray finding showed bilateral lung infiltrate with normal heart size as shown in Figure 1.

Table 1. Laboratory Examination

\begin{tabular}{|c|c|c|}
\hline Test & Result & Reference \\
\hline Hemoglobin & 10.4 & $11.0-13.0 \mathrm{mg} / \mathrm{dL}$ \\
\hline Hematocrite & 30.7 & $40-50 \%$ \\
\hline Leucocyte & 4.550 & $5.000-10.000 / \mu \mathrm{L}$ \\
\hline Trombocyte & 151.000 & $150.000-400.000 / \mu \mathrm{L}$ \\
\hline MCV/MCH/MCHC & $83.5 / 28.2 / 33.8$ & \\
\hline Diff count & 0/0/76/21/3 & \\
\hline D-dimer & 500 & $0.0-0.3 \mathrm{mg} / \mathrm{dL}$ \\
\hline Fibrinogen & 302.2 & $150-400 \mathrm{mg} / \mathrm{dL}$ \\
\hline APTT & $36.4(35.1)$ & $31-47.0(\mathrm{~s})$ \\
\hline PT & $9.2(10.4)$ & $9.8-11.2(\mathrm{~s})$ \\
\hline Albumin & 3.10 & $3.4-4.8 \mathrm{~g} / \mathrm{dL}$ \\
\hline Globulin & 3.79 & $1.8-3.9 \mathrm{~g} / \mathrm{dL}$ \\
\hline Total protein & 6.89 & $6.4-8.7 \mathrm{~g} / \mathrm{dL}$ \\
\hline SGPT (ALT) & 2 & $0-49 \mathrm{u} / \mathrm{L}$ \\
\hline SGOT (AST) & 126 & $0-32 \mathrm{u} / \mathrm{L}$ \\
\hline Uric acid & 7.4 & $0-6.9 \mathrm{mg} / \mathrm{dL}$ \\
\hline Creatinine & 0.559 & $0.8-1.3 \mathrm{mg} / \mathrm{dL}$ \\
\hline Ureum & 28.3 & $0-49 \mathrm{mg} / \mathrm{dL}$ \\
\hline Natrium & 142 & $132-147 \mathrm{mEq} / \mathrm{L}$ \\
\hline Kalium & 3.4 & 3.3-5.4 mEq/L \\
\hline Clorida & 112 & 94-111 mEq/L \\
\hline $\mathrm{LDH}$ & 569 & $<2155 \mathrm{U} / \mathrm{L}$ \\
\hline GDS & 92 & $0-200 \mathrm{mg} / \mathrm{dL}$ \\
\hline HBsAg & Non reaktif & Non reaktif \\
\hline CRP & 40 & $<5 \mathrm{mg} / \mathrm{L}$ \\
\hline Anti HCV & Non reaktif & Non reaktif \\
\hline Microalbuminuria & \multicolumn{2}{|l|}{$67.5 \mathrm{mg} / 24$ hours } \\
\hline \multicolumn{3}{|l|}{ TB Screening } \\
\hline IGRA & \multicolumn{2}{|l|}{ Unremarkable } \\
\hline Gene Xpert & \multicolumn{2}{|l|}{ Negative } \\
\hline AFB smears 3 times & \multicolumn{2}{|l|}{ Negative } \\
\hline BGA & \multicolumn{2}{|c|}{ 7.5/26.1/100/24.1/-2.5/98.7 } \\
\hline \multicolumn{3}{|l|}{ Autoimune Analysis } \\
\hline ANA & \multicolumn{2}{|c|}{ Positive 1/1000 (homogen) } \\
\hline $\begin{array}{l}\text { B2GP-IgM } \\
\text { B2GP-IgG }\end{array}$ & $\begin{array}{l}21.2 \mathrm{U} \\
4.3 \mathrm{U}\end{array}$ & $\begin{array}{l}<20 \mathrm{U} \\
<20 \mathrm{U}\end{array}$ \\
\hline Lupus anticoagulant & Weak positive & negative \\
\hline
\end{tabular}
Anti-ds DNA
9.4
$<100 \mathrm{IU} / \mathrm{mL}$
CK
ACA IgM
ACA IgG
$58 \mathrm{U} / \mathrm{L}$
$<192 \mathrm{U} / \mathrm{L}$
26.4 low to medium positive
25.1 low to medium positive

$\begin{array}{lll}\text { C3 } & 6 & 90-180 \mathrm{mg} / \mathrm{dL} \\ \text { C4 } & 16 & 10-40 \mathrm{mg} / \mathrm{dL} \\ \text { Rheumatoid Factor } & 12 & <14 \mathrm{U} / \mathrm{ml}\end{array}$

Figure 1. Chest X-ray

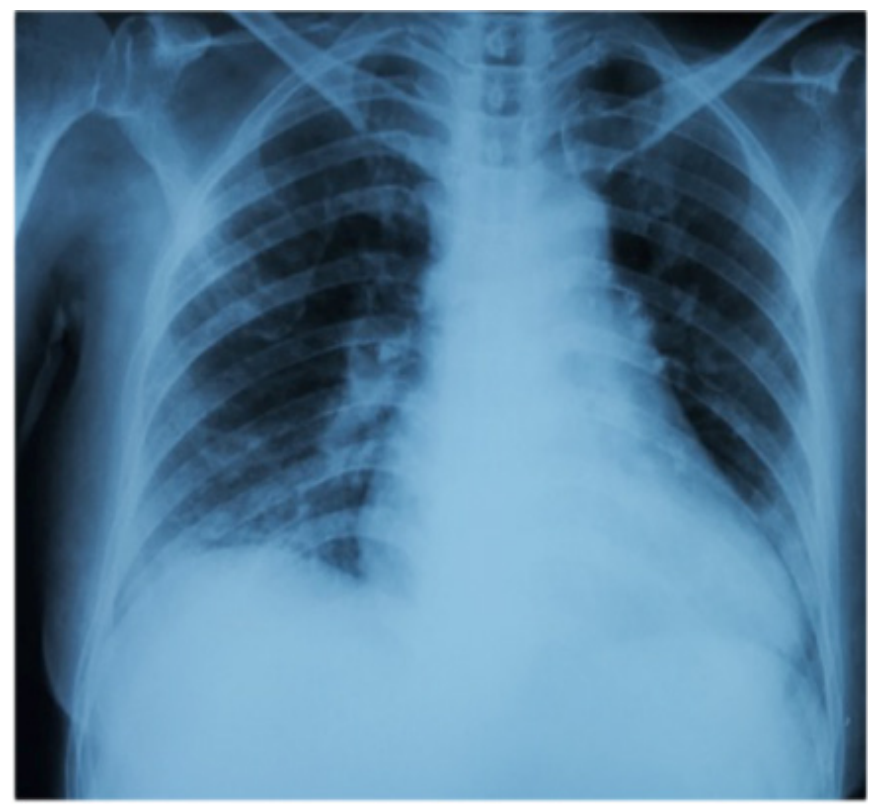

Note: Bilateral infiltrate in both lungs' base.

Patient was initially diagnosed as Health Care Associated Pneumonia (HCAP), MCTD with possible ILD, and anemia. She treated with cefepime $2 \times 1$ gr/IV, N-acetylcysteine $1 \times 600 \mathrm{mg} / \mathrm{PO}$, and nebulization with salbutamol $3 \times 2.5 \mathrm{mg}$. MTX were given subsequently and increased to $1 \times 10 \mathrm{mg} /$ $\mathrm{PO} /$ week. Other medications, included: cilostazol $2 \times 50 \mathrm{mg} /$ $\mathrm{PO}$, omeprazole $1 \times 20 \mathrm{mg} / \mathrm{PO}$, folic acid $5 \mathrm{mg} / \mathrm{PO} /$ weeks, nifedipine $1 \times 10 \mathrm{mg} / \mathrm{PO}$ and MP $3 \times 4 \mathrm{mg} / \mathrm{PO}$ were continued.

Chest CT-scan shown a typical finding for ILD with possible undifferentiated interstitial pneumonitis (UIP) or nonspecific interstitial pneumonitis (NSIP) (Figure 2). Spirometry showed a restrictive pattern with low FEV1: $1.26 \mathrm{~L}$ or $40.6 \%$ predicted. Echocardiography showed: a normal ejection fraction (EF) $62 \%$ with no signs of pulmonary hypertension. Skin biopsy resulted consistent with scleroderma. Bone surveys showed: signs of multiple erosion and osteopenia. Electromyograph revealed carpal tunnel syndrome over left arm and myotonic lesion over the lower extremities. Then patient treated with fluticasone - salmeterol $500 \mathrm{mcg} / 50 \mathrm{mcg}$ two times daily attenuation, oral cavit D3 thrice daily, oral zolendronic $35 \mathrm{mg} /$ weekly, she was planned to undergo cyclophosphamide pulse dose $500 \mathrm{mg} / \mathrm{IV}$. First dose of intravenous cyclophosphamide $500 \mathrm{~m} \mathrm{mg}$ was started as the clinical condition improved, and continued every two weeks until 6 doses given. 


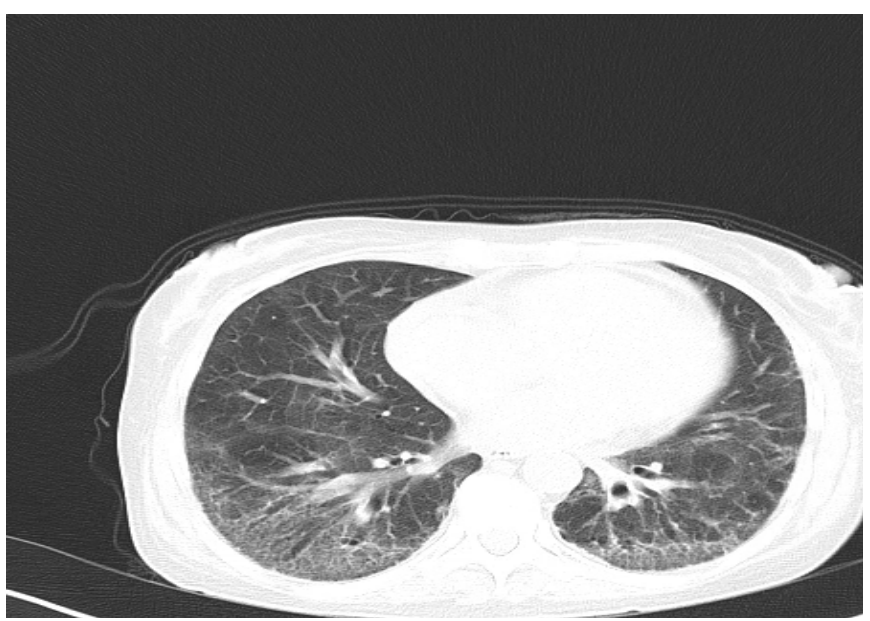

Figure 2. Patient Chest CT-scan

Note: Chest CT-scan showed ground glass opacity and traction bronchiectasis in subpleura and both lung base (arrow sign); multiple lymphadenopathies of paratrakeal and sub carinal.

Unfortunately, after 1 month of treatment, she readmitted due to the progression of dyspnea. The clinical findings notably showed the progression of ILD, but the clinicians denied to give further dose of cyclophosphamide. She was hospitalized and developed sepsis due to hospital acquired pneumonia (HAP) in the fourth day of hospitalization. She got treatment contained intravenous meropenem $3 \times 1$ gr daily and intravenous levofloxacin $500 \mathrm{mg}$ daily. However on the $8^{\text {th }}$ day,she pronounced death due to irreversible septic shock.

\section{MCTD and pulmonary involvement}

MCTD was first described by Sharp et al (1971- 72) as a distinct syndrome with similar features of systemic lupus erythematosus (SLE), systemic sclerosis (SSC), dermatomyositis/polymyositis and rheumatoid arthritis (RA). The disease was associated with autoantibodies to ribonuclease - sensitive component of extractable nuclear antigen (U1 RNP). ${ }^{7}$ It is a rare disease and affected only 2.7/1.000.000 patients in Japan population. Various studies show the correlation between the disease and the existence of HLA-DR4 and HLA-DR2. The clinical symptoms of MCTD usually takes several years before the diagnosis of MCTD established. Diagnosis of MCTD can be made using criteria from Alarcon-Segovia and Kahn which have sensitivity $62.5 \%$ and specificity $86.2 \% .{ }^{8}$ Our patients showed moderate positive U1 RNP, Reynaud's phenomenon, acrosclerosis, history of synovitis, and possible myositis hence satisfy the diagnosis of MCTD.

The presence of pulmonary involvement occurs in $75 \%$ patients. ${ }^{8}$ Common pulmonary problems could be found, such as: pleural effusion $(50 \%){ }^{10}$ pleuritic pain, pulmonary hypertension, ILD, thromboembolic disease, alveolar hemorrhage, diaphragmatic dysfunction, aspiration pneumonitis/pneumonia, obstructive airway disease, pulmonary infections, pulmonary vasculitis. ${ }^{7}$ Vegh, et al reported from 179 patients with MCTD, 96 patients (53.6\%) had ILD, with onset approximately 2-4 years prior to the presence of the symptoms. ${ }^{11}$ which confirmed common interstitial pneumonitis. The patients were followed-up, and the data of computed tomography and respiratory function tests were detected 6 months, and then 4 years after the acute lung disease complicated by mixed connective tissue disease. RESULTS: Out of the 179 mixed connective tissue disease patients 96 (53.6\% Meanwhille Gunnarsson, et al reported that abnormal pulmonary function tests (PFTs) restrictive pattern were seen in up to $90 \%$ of patients with MCTD. ${ }^{13}$ This finding is compatible with our patient.

\section{Pathophysiology ILD in MCTD}

The role of autoimmunity in ILD associated with CTD is well established. The higher concentration of proinflammatory and profibrotic mediators have been implicated in the pathogenesis of ILD and IPF, and might also have important roles in SSc-associated ILD. Those mediators include chemokines, cytokines, growth factors, lipids, and prostanoids. The pivotal mediator of fibrosis is the multifunctional cytokines, transforming growth factor beta (TGF $\beta$ ). Substantial evidences implicate that TGF $\beta$ - along with platelet-derived growth factor, endothelin-1 (ET-1), and other cytokines - plays role in the pathogenesis of SSc. ${ }^{3}$ (Figure 3).

Figure 3. Pathogenesis ILD and pulmonary fibrosis in MCTD. ${ }^{3}$

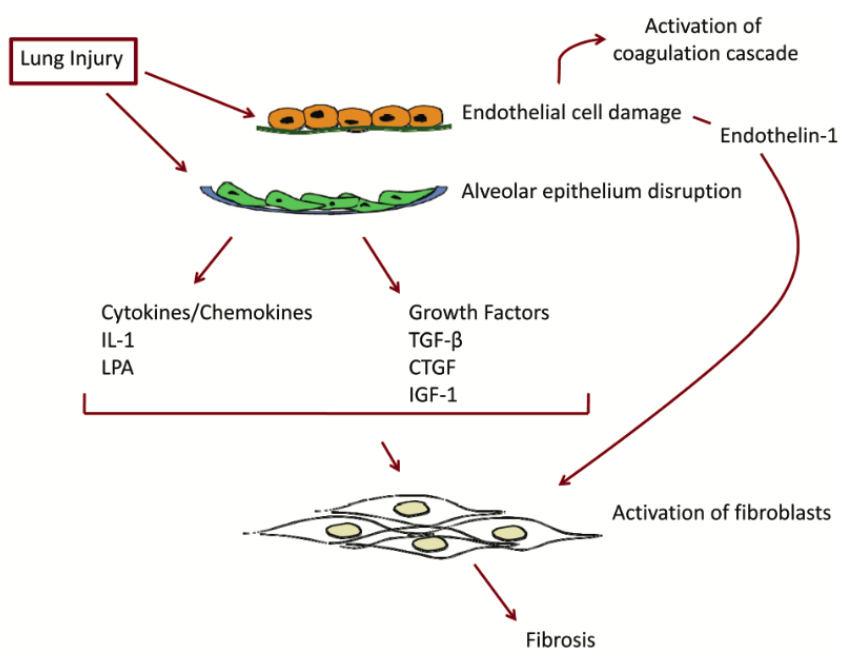

CTGF: connective tissue growth factor; IGF-1: insulin-like growth factor-1; LPA: lysophosphatidic acid; TGF- $\beta$ : transforming growth factor beta

Pathologic findings of pulmonary involvement of MCTD are classified into interstitial fibrosis and vascular changes. ${ }^{15}$ Interstitial fibrosis typicaly shows appearance as UIP. It causes distortion of the alveolar architecture. On the other hand, vascular changes are typicaly consisted of bland intimal proliferation of the lung arterioles, ${ }^{10}$ plexiogenic angiopathy, and chronic pulmonary emboli, which subsequently cause pulmonary hypertension. ${ }^{8}$

\section{Approach to patient with ILD in CTD}

As discussed above pulmonary manifestation may vary in MCTD. A comprehensive history assesment may provide valuable informations lead to certain diseases, include CTD- 
ILD. Differential diagnosis can be narrowed when the history and clinical findings combined with appropriate measurements of lung function and specific blood tests such as autoimmune serologies consistent to CTD. Moreover, if suspected extrapulmonary tissue sampling (e.g. lymph node or skin biopsy) and thoracic imaging can be performed. Clinical approach for diagnosing ILD-CTD is represented in Figure 4. Assesing spesific cause of ILD may needed invasive diagnostics (bronchoscopy, biopsy and bronchoalveolar lavage).

Figure 4. Approach to the diagnosis in ILD-CTD ${ }^{16}$

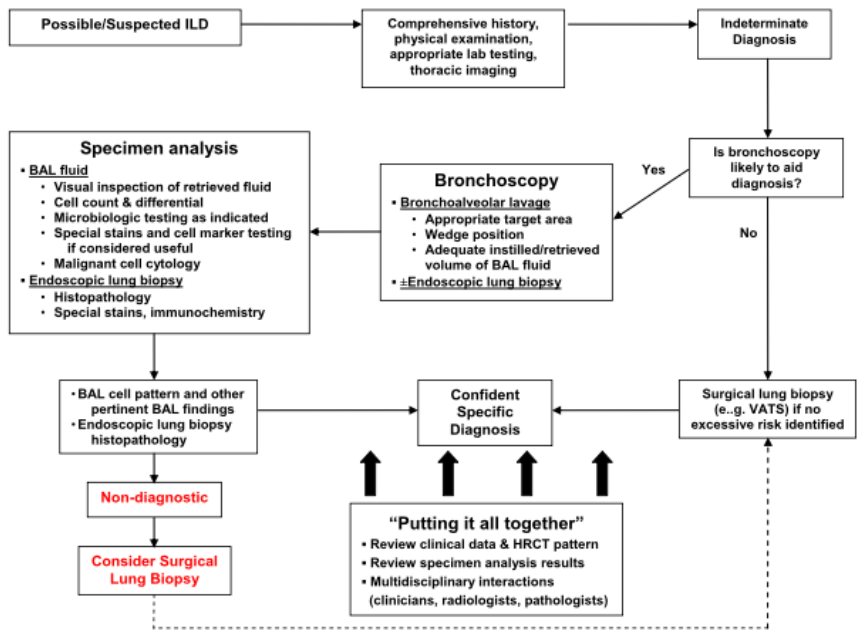

BAL: bronchoalveolar lavage fluid, HRCT:- High resolution computed tomography; ILd: Interstitial lung disease; VATS: video assisted thoracoscopic surgery.

When ILD is suspected, chest X-ray is recommended to be performed in two projections posterior-anterior and lateral view. Chest X-ray can show an interstitial pattern in $19 \%$ of population. The other findings consist of irregular linear hyperattenuating areas with a reticular pattern and involving mainly the lung bases. ${ }^{15}$ In the late stage, honeycomb apperance may be identified.

High Resolution-CT (HRCT) of the chest is the most sensitive test in establishing ILD diagnosis. ${ }^{8}$ In previous study, ILD was detected in $48 \%$ of MCTD patients using HRCT. ${ }^{17}$ often serious, manifestation of mixed connective tissue disease (MCTD However, HRCT result should be supported by clinical symptoms and histopatological results. Because, in some cases it may mimic the other diseases, such as: NSIP and UIP. Study by Kosuka, et al found that groundglass attenuation almost identified in all ILD patients. Other frequent findings seen by HRCT were intralobular reticular opacity, traction bronchiectasis, honeycombing, subpleural small nodules, and non-septal linear opacity predominant in the lower and peripheral lung fields. ${ }^{15}$ Radiologic abnormalities include areas of parenchymal consolidation that may be related to BOOP (bronchiolitis obliterans organizing pneumonia). ${ }^{18}$

Bronchoscopy and/or surgical lung biopsy may be required to make a more convince diagnose of specific ILD. ${ }^{6}$ The right middle lobe or lingula of the left upper lobe are likely to be the best regions to perform lavage when diffuse disease is present. Areas with ground-glass opacification or profuse nodular change are more likely to provide useful diagnostic information. ${ }^{16}$ Endobronchial biopsy provide useful information if endobronchial abnormalities are present (e.g. superficial nodules, mucosal ulceration). Trans bronchial lung biopsy is best performed far from the area of advanced fibrosis. A surgical lung biopsy (SLB) obtained via videoassisted thoracic surgery (VATS) or open biopsy is likely to provide an excellent specimen. ${ }^{16}$

The main histopatologic changes of pulmonary involvement in MCTD is classified into interstitial fibrosis and vascular changes. Interstitial fibrosis has the appearance of usual or NSIP. Typical vascular changes consist of bland intimal proliferation of the lung arterioles, plexogenic angiopathy and chronic pulmonary emboli. ${ }^{18}$ Histopatologic examination could be used to confirm the presence of ILD however the results may vary. Most patient ILD due to MCTD shows histopatologic pattern of NSIP followed by UIP. ${ }^{3}$

\section{Treatment ILD-MCTD}

The general strategies recommended for managing idiopathic pulmonary fibrosis (IPF) are often applied in CTD-ILD. These include the use of supplemental oxygen in patients with resting hypoxemia and treatment of asymptomatic gastroesophageal reflux disease (GERD). ${ }^{4}$ There are no specific guidelines exist for managing acute exacerbations of ILD in CTD. Interventions commonly give, included: Broad spectrum antimicrobials, coverage for pneumocystis jirovecii and fungal is considered based on risk factors. Risk factor assessed, such as preexisting immunosuppression, removal of the offending agents if drug toxicity suspected. High doses of pulse methylprednisolone (1g IV daily for three days) and IV or oral cyclophosphamide is considered. ${ }^{4}$

There is a few published literatures regarding the correlation of histology findings in ILD associated with MCTD and the use of immunosupresive therapy. Administration of immunosupressive therapy, such as corticosteroid, DMARD, or others immunosuppressive drugs showed no significant evidence for a better outcome. ${ }^{10}$ However, we found one study which showed $47 \%$ of patients with MCTD-ILD respond better to $2 \mathrm{mg} / \mathrm{kg}$ /day corticosteroids. ${ }^{17} \mathrm{often}$ serious, manifestation of mixed connective tissue disease (MCTD On the first admission our patient got broad spectrum antibiotic, cefepime $2 \times 1 \mathrm{gr} / \mathrm{IV}$, after that she treated with intravenous cyclophosphamide pulse therapy.

Other consideration in treating ILD is assessing any other comorbid conditions. ${ }^{4}$ For example pulmonary hypertension $(\mathrm{PH})$. It is diagnosed if mean pulmonary artery pressure found $\geq 25 \mathrm{mmHg}$. Therefore it can only be diagnosed by right heart catheterization. Therapy for PAH is quite specific, it is included anticoagulation, oxygen, diuretics, and pulmonary vascular vasodilators, phosphodiesterase inhibitor, imunosupressant. ${ }^{10}$

\section{Conclusion}

Pulmonary manifestations of MCTD patients may vary from mild to severe respiratory symptoms. The hallmark of pulmonary manifestation in MCTD is vasculopathy and $\mathrm{PH}$ 
which manifestated as progressive dyspnea, chronic cough, and even hemoptysis. A history of progressive dyspnea and typical HRCT findings is required in order to establish the diagnosis of ILD-MCTD. There was no specific recommended guidelines for the treatment of ILD-MCTD. Several case reports shown the improved of patient's survival when using pulse dose corticosteroid and immunosuppressant. Some patients only respond to high dose corticosteroid.

\section{REFFERENCE}

1. Capobianco J, Grimberg A, Thompson BM, et al. Thoracic Manifestations of Collagen Vascular Diseases. RadioGraphics 2011; 32: 33-50.

2. American Thoracic Society, European Respiratory Society. American Thoracic Society / European Respiratory Society International Multidisciplinary Consensus Classification of the Idiopathic Interstitial Pneumonias. Am J Respir Crit Care Med. 2002; 165 (2): 277-304.

3. Castelino FV, Varga J. Interstitial lung disease in connective tissue diseases : evolving concepts of pathogenesis and management. Arthritis Res Ther. 2010; 12(4): 213-224.

4. Mathai SC, Danoff SK. Management of interstitial lung disease associated with connective tissue disease. BMJ. 2016; 352: h6819.

5. Fischer A, Antoniou KM, Brown KK, et al. An official European Respiratory Society / American Thoracic Society research statement: interstitial pneumonia with autoimmune features. Eur Respir J. 2015; 46(4):976987.

6. Travis WD, Costabel U, Hansell DM, et al. An official American Thoracic Society/European Respiratory Society statement: Update of the international multidisciplinary classification of the idiopathic interstitial pneumonias. Am J Respir Crit Care Med 2013; 188(6): 733-748.
7. Narsimulu G. Mixed connective tissue disorders (mctd). Med Updat 2010; 17: 842-845.

8. Bennet R. Overlap syndromes. In: Kelley's Textbook of Rheumatology Garry S F, Ralph C B, Harris Jr ED, et al. (eds). USA: Saunders; 2017: 1489-1511.

9. Bodolay E, Szekanecz Z, Vegh J, et al. Mixed connective tissue disease: should the diagnosis be more restrictive?: reply. Rheumatol 2005; 44: 1466-1467.

10. Bull TM, Fagan KA, Badesch DB. Pulmonary Vascular Manifestations of Mixed Connective Tissue Disease. Rheum Dis Clin 2017; 31: 451-464.

11. Vegh J, Szilasi M, Soos G, et al. Interstitial lung disease in mixed connective tissue disease. Orv Hetil 2005; 146: 2435-2443.

12. Colin G, Nunes H, Hatron PY, et al. Étude Des Pneumopathies Interstitielles Diffuses De La Connectivite Mixte. Rev Mal Respir 2010; 27: 238-246.

13. Gunnarsson R, Aaløkken TM, Molberg Ø, et al. Prevalence and severity of interstitial lung disease in mixed connective tissue disease: a nationwide, cross-sectional study. Ann Rheum Dis 2012; 1966-1972.

14. Jindal SK, Agarwal R. Autoimmunity and interstitial lung disease. Curr Opin Pulm Med 2005; 11: 438-446.

15. Kozuka T, Johkoh T, Honda 0, et al. Pulmonary involvement in mixed connective tissue disease: high-resolution CT findings in 41 patients. $J$ Thorac/maging 2001; 16: 94-98.

16. Meyer KC. Diagnosis and management of interstitial lung disease. Trans/ Respir Med 2014; 2: 1-13.

17. Bodolay E, Szekanecz Z, Dévényi $K$, et al. Evaluation of interstitial lung disease in mixed connective tissue disease (MCTD). Rheumatol 2005; 44: 656-661.

18. Kim EA, Lee KS, Johkoh T, et al. Interstitial Lung Diseases Associated with Collagen Vascular Diseases: Radiologic and Histopathologic Findings. RadioGraphics 2002; 22: S151-S165. 
